\title{
CRENÇAS dAS MÃeS EM RELAÇÃo A VACINAÇÃo
}

\author{
Silvia Lúcia Ferreira1
}

\begin{abstract}
RESUMO. O objetivo deste trabalho foi identificar a importância das variáveis do Modeio de Crenças em Saúde no Comportamento das mães que iniciam a vacinação básica dos filhos. Segundo este Modelo, para uma pessoa adotar um determinado comportamento preventivo em saúde ela precisa acreditar: a) que é suscetível à doença; b) que a doença é grave; c) que existem muitos benefícios e pouca ou nenhuma barreira para a ação; e d) que haja um evento motivador que direcione a ação a ser tomada. Após verificar como essas variáveis se comportam em relação à amostra, concluiu.se que os resultados são compatíveis com o Modelo utilizado.
\end{abstract}

ABSTRACT. The goal of this article is to identify the variables of beliefs model In health of mothers who started the basic vaccination of their chidren. According to this model people to follow the preventive measures need to know: 1) person is susceptible to disease; 2) disease is serious; 3 ) there are many advantages and few or no barriers to action; and 4) a motivation must be present to guide the action. After collecting data and analysis, the results show that they are compatible to the used model.

Nos últimos cinquienta anos, a vacinação teve sucesso excepcional em países desenvolvidos, no controle da difteria, coqueluche, tétano, poliomielite, sarampo e tuberculose, diminuindo os índices de mortalidade infantil. Nos países da América do Norte, Europa, Rússia e Oceania a taxa de mortalidade na faixa de um a quatro anos é de uma para cada mil crianças nascidas vivas (OPAS/OMS, 1979).

Nos países em vias de desenvolvimento essa taxa ainda é muito elevada: a cobertura vacinal nestes países é baixa, nem sequer $10 \%$ dos 80 milhões de crianças que nascem anualmente têm acesso aos recursos da imunização.
Das seis doenças infecto-contagiosas mencionadas, as que causam maior mortalidade são o sarampo, a coqueluche e o tétano. As duas primeiras, que ceifam a vida de crianças não imunizadas, têm percentagens de casos mortais compreendidas entre 1 e $10 \%-0$ dado mais alto ocorre entre as crianças menores e mais mal nutridas - o tétano neunatal afeta provavelmente menos de $2 \%$ das crianças nascidas nos países em desenvolvimento, de mães sem imunização, porém mata de 70 a $90 \%$ das crianças atingidas pela doença. A morbidade e mortalidade causadas pela difteria são muito difíceis de determinar com pre-

1. Pós-Graduação em Enfermagem. Universidade Federal de Santa Catarina, Florianópolis, SC. 
cisão, mas se estima que a doença causa anualmente mais de cem mil mortes entre as crianças menores de cinco anos (OPAS/OMS, 1979).

Para MAHLER (1977) esta situação de saúde está relacionada aos seguintes aspectos: 1) o público e os profissionais de saúde não se dão conta dos custos dessas doenças em termos de saúde e de vidas, nem de como tem sido eficaz a vacinação para diminuir este custo; 2) não existe um contato satisfatório entre os serviços de saúde e as mães para que as vacinas sejam aplicadas na idade correta; 3) os requisitos técnicos de um programa nacional de vacinação exigem técnicas de planejamento, administração e supervisão no campo da prática que podem não ter sido ainda adquiridos pelos serviços básicos de saúde. Por outro lado, CASTRO (1981) argumenta que, apesar de ter havido um avanço dos conhecimentos científicos na prevenção das moléstias infecto-contagiosas como raios $\mathrm{X} m$ massa, vacinações e outros, e um crescimen $\rho$ rápido e desordenado das cidades, não houve, nos países em desenvolvimento, avanço correspondente na infra-estrutura dos serviços básicos à população. A deficiência ou inexistência de suprimento de água, canalização de esgotos, coleta de lixo, acrescida das péssimas condições de higiene, habitação, alimentação e trabalho, propiciaram o surgimento de epidemias com aumento das taxas de morbidade e mortalidade. Em todas as capitais brasileiras, 0 índice de mortalidade infantil atinge níveis assustadores, traduzindo as péssimas condições de vida da população em geral e das crianças em particular. Recife e Teresina atingiram, em 1974, respectivamente os índices de mortalidade de 256 e 90 por 1.000 crianças nascidas vivas (CENTRO DE DEFESA DA QUALIDADE DE VIDA, 1979).

Além de toda esta infra-estrutura sócio-econômica determinando os níveis de saúde das populações, ressaltem-se algumas características dos programas de saúde: os programas de controle de doenças transmissíveis nos países da América Latina foram, por muito tempo, dirigidos às enfermidades endêmicas que podiam causar morte ou incapacitar a força de trabalho economicamente produtiva. No Brasil, estes programas concentraram-se no combate às endemias e saneamento dos portos e núcleos urbanos mais importantes como São Paulo, Rio de Janeiro e Santos. Estes núcleos estavam ligados às atividades de comércio, financiamento, transporte e exportação cafeeira. Além disso, havia necessidade de se proporcionarem boas condições sanitárias locais para as relações comerciais com $o$ exterior e para atrair imigrantes que constituiriam a mão de obra indispensável à formação do mercado de trabalho capitalista.

Vários outros planos inicialmente propostos não tiveram sucesso, como afirma MELO (1981), basicamente, por não levar-se em conta outras características, dentre as quais a falta de estudo das variáveis de natureza econômica, desconhecendo as diferenças regionais, ignorando-se a diversidade dos problemas urbanos e rurais, omitindo-se a importância da desigualdade de distribuição social e demográfica da renda, esquecendo-se a dependência do setor de saúde em relação a todos os demais setores de atividades sociais e econômicas.

Moldado com as mesmas características dos outros programas de saúde, surgem as campanhas de imunização contra febre amarela, malária e varíola, em 1904. Somente a partir de 1975, com a Lei 7.259, o Programa Nacional de Imunizações torna-se obrigatório, ligado ao recebimento do salário família (MINISTÉRIO DA SAÚDE, 1976).

Atualmente, também a aceitação do fato de que simultaneamente podem administrar-se antígenos de vacinas múltiplas, sem reduzir de maneira significativa a proteção, aliada à obrigatoriedade, tem permitido aumentar a cobertura através de esquemas simplificados. Mesmo assim observa-se que apesar da crescente melhoria na percentagem de crianças que receberam a vacina tríplice (DPT) este total representa apenas $50 \%$ da população infantil estimada para 1979. Para a vacina Sabin o índice atingido em relação à mesma população foi de 48,6\% (MINISTÉRIO DA SAÚDE, 1980).

Estes índices nos levam a crer que devem existir vários fatores ligados a esta situação. Por que, apesar de existirem vacinas grátis, programas estruturados e campanhas de vacinação, os índices de doenças infecto-contagiosas preveníveis através da imunoprofilaxia continuam altos?

Para ROSENSTOCK (1974), todo programa de saúde que precisa obter a cooperação do cliente, independente do mesmo estar assintomático, com alguns sintomas ou mesmo doente, leva a algumas perguntas de como o povo se comporta, 0 porquê deste comportamento e como ele pode ser modificado.

O comportamento preventivo em saúde é evidenciado através da prática de atividades que $o$ indivíduo acredita serem benéficas para se manter sadio. Essas atividades poderão prevenir a doença ou detectá-la em um estágio 
assintomático. O comportamento em saúde, como todo comportamento humano, é imensamente complexo. Um simples ato de escovar os dentes ou tomar um analgésico para dor de cabeça é o resultado de influências passadas e presentes provenientes de várias fontes. HOCHBAUM (1974) afirma que o comportamento em saúde é motivado primariamente por um desejo de proteger-se contra as ameaças à sua saúde e segurança. Importa pouco se uma ameaça é real ou imaginária, porque o homem age não de acordo como as coisas são, mas de acordo como ele percebe as coisas. Para se tomar uma decisão particular em saúde vai depender se a pessoa percebe ou não uma doença como ameaça.

Apesar da percepção desta ameaça, na prática, ao analisarmos o processo de tomada de decisões em saúde, constatamos que existe uma inconsistência entre os conhecimentos (o que se sabe), as atitudes (o que se sente), e a prática ou comportamento preventivo (o que se faz). Para CANDEIAS (1979) é comum encontrarmos indivíduos que tiveram acesso a conhecimentos científicos, consideram-nos relevantes para a manutenção da saúde mas adotam um comportamento inconsistente.

$\mathrm{Na}$ nossa prática profissional seguidamente surge uma questão: Por que nem todas as mães vacinam os filhos? E possível que existam fatores ligados ao comportamento que funcionem como barreiras para a ação, quando fatores situacionais, como acesso aos serviços de saúde, custo e outros, não existem.

Parece-nos, pois, fundamental que estudos na área do comportamento em saúde sejam realizados para que possam surgir propostas científicas de modelos comportamentais adaptados à nossa realidade e que estes modelos, quando aplicados, forneçam uma base teórica para os programas de educação em saúde.

Procurando uma possível resposta para nossa questão, iniciamos por tentar entender o comportamento das mães que levam os filhos para vacinar. Quais as crenças que estas mães possuem sobre vacinação que interferem de forma positiva para que as vacinas sejam aplicadas? Optamos por aplicação de um modelo comportamental, porque entendemos que para uma teoria comportamental ser de valor para a Saúde Pública ela deve ser testada em situação real onde sofre intervenção de fatores situacionais. A maior contribuição da prática é a operacionalização dos conceitos. Outro motivo que interferiu na nossa opção foi o fato da não terem sido encontrados na revisão bibliográfica, trabalhos feitos no Brasil que utilizaram modelos comportamentais para fundamentar a prática de saúde.

Este trabalho está baseado na teoria do Comportamento Preventivo de ROSENSTOCK (1974). O autor propõe o Modelo de Crenças em Saúde (MCS) para operacionalizar a teoria.

O Modelo (MCS) em termos de dimensão inclui duas classes variáveis: 1) o estado psicológico de alerta para agir (suscetibilidade e gravidade percebidas) e 2) o grau com que uma ação é acreditada como benéfica para reduzir a ameaça à saúde (benefícios menos barreiras e eventos motivadores).

Entendemos que os resultados deste estudo não poderão ser generalizados para as mães que não vacinam seus filhos, mas é possível que ele ofereça algum subsídio para um melhor entendimento do comportamento dessas mães.

\section{OBJETIVOS}

Identificar a importância das variáveis do Modelo de Crenças em Saúde no comportamento preventivo das mães que iniciam a vacinação básica dos filhos.

\section{SUPORTE TEÓRICO}

A teoria do Comportamento em Saúde foi desenvolvida na década de 1950 . O propósito desta teoria foi criar um suporte para tentar explicar o comportamento relacionado à saúde e unificar as descobertas, ainda não relatadas, sobre outras investigações na área.

O Modelo de Crenças em Saúde (MCS) de Irvin Rosenstock descreve algumas variáveis que contribuíram significativamente para 0 entendimento do comportamento na área da saúde. Este modelo inclui duas classes de variáveis: 1) o estado psicológico de alerta para agir; 2) o grau com que uma determinada ação é acreditada como benéfica para reduzir a ameaça à saúde. As variáveis que compõem o Modelo são as seguintes:

1) Suscetibilidade percebida: Predisposição interior ou risco objetivo de contrair uma determinada condição. Existem vários níveis de suscetibilidade para cada indivíduo relacionado às doenças. Existem pessoas que não aceitam qualquer possibilidada de contrair uma doença, os que se vêem constantemente. ameaçados e os que acreditam que a sua sus. cetibilidade pessoal é variável.

2) Gravidade percebida: - A gravidade pode ser julgada pelo grau de comprometimen- 
to emocional criado pela força da doença, como também pelos tipos de dificuldades que 0 indivíduo acredita que uma determinada condição de doença lhe causará. Um indivíduo pode perceber $\mathrm{o}$ problema em termos de conseqüências médicas ou clínicas como redução das suas funções físicas ou mentais por um longo período de tempo ou incapacidade permanente. A gravidade percebida, para outros, pode incluir outras implicações tais como os efeitos da doença no seu trabalho, vida familiar e relações sociais.

3) Beneficios e barreiras percebidas para ação: - A aceitação de suscetibilidade e gravidade a uma doença produz uma força levando à ação, mas não define o curso particular da ação a ser tomada. Uma alternativa será considerada benéfica se conduzir a uma redução de suscetibilidade e gravidade de uma determinada doença. Por outro lado, um indivíduo pode crer que uma determinada ação é efetiva para reduzir a ameaça da doença, mas ao mesmo tempo pode percebê-la como inconveniente, cara, desagradável, dolorosa. Esses aspectos negativos servem como barreiras e criam motivos para que estas ações sejam evitadas. Se o estado de alerta para a ação é alto e os aspectos negativos são percebidos como baixos a ação é tomada. Se ao contrário, o estado de alerta é baixo e os aspectos negativos são percebidos como fortes, estes funcionam como barreiras e evitam que a ação seja tomada.

4) Eventos motivadores: - Os níveis de suscetibilidade, gravidade, barreiras e benefícios percebidos geram energia e força para a ação. Entretanto, estas combinações podem não resultar em ação, a não ser que ocorra algum evento estimulador que ponha em movimento o processo. $\mathrm{Na}$ área da saúde, tais eventos ou caminhos podem ser internos (percepção do estado corporal) e externos (impacto dos meios de comunicação, recebimento de alguma notícia de doença e/ou orientação de profissionais da saúde).

A intensidade requerida por estes eventos para acionar um comportamento preventivo varia de acordo com os níveis de alerta. Um estado de alerta baixo, devido à baixa aceitação de suscetibilidade e gravidade, necessita de estímulo mais intenso. Ao contrário, se existe alta prontidão ou alerta para a ação, o estímulo necessário para acionar um comportamento preventivo é baixo.

A Figura 1 sumariza o Modelo de Crenças em Saúde (MCS).

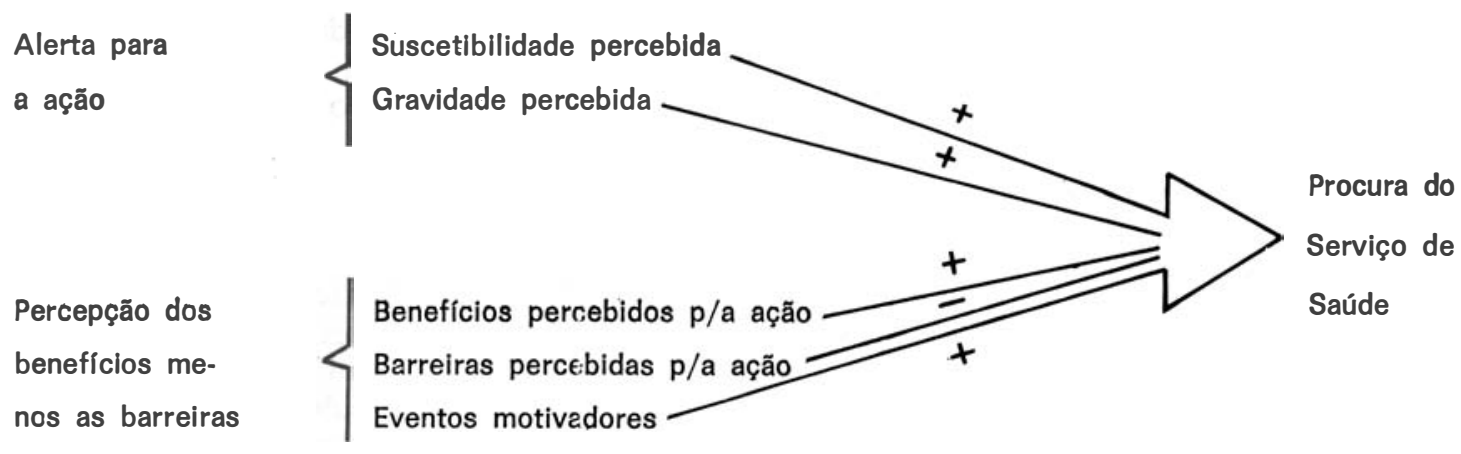

Figura 1. Representação esquemática dias variáveis do Modelo de Crenças em Saúde de Rosenstock (traduzido e transcrito sem autorização da autora). In: BARRET, M. B. The health behavior of persons utilizing diagnostic cancer screening. Chicago, $1979: 20$.

\section{METODOLOGIA}

\section{Tipo de estudo}

Este é um estudo exploratório-descritivo que visa identificar a importância das variáveis do Modelo de Crenças em Saúde (MCS) no comportamento preventivo de mães que iniciam a vacinação básica dos filhos, como também identifica a relação da renda mensal familiar e nível de escolaridade dessas mães com as variáveis do modelo.

\section{Local do estudo}

O presente estudo foi realizado no Centro de Saúde da cidade de Florianópolis, SC. 


\section{População e amostra}

A população deste estudo foi composta por mães que levam seus filhos para iniciarem a vacinação básica aos dois meses de idade. A amostra foi obtida durante os meses de setembro e outubro de 1982, composta de oitenta sujeitos. O tipo de amostragem foi não probabilística propositada.

\section{Instrumento para coleta de dados}

Optou-se por desenvolver um instrumento com base no modelo teórico, formulando-se questões para medir cada variável do referido Modelo.

\section{Procedimentos para aperfeiçoamento do instrumento de coleta de dados}

O instrumento Crenças das Mães em Relação à Vacinação (CMRV), após ser modificado cinco vezes porque não atendia aos objetivos propostos, foi aplicado a quatro mães visando testar a adequação da linguagem e clareza dos itens. Após feitas as modifica: ções, o instrumento foi enviado aos peritos para validação aparente e de conteúdo.

\section{Validação aparente e de conteúdo}

Para participar da validação aparente e de conteúdo do instrumento (CMRV), foram convidados treze peritos, todos enfermeiros, com experiência em Saúde Pública. Foi estipulado um nível de concordância de $80 \%$ entre os validadores para que uma questão permanecesse no instrumento.

\section{Confiabilidade do instrumento}

Para determinar a confiabilidade do instrumento foi utilizado o método de consistência interna sendo calculado o coeficiente alfa de Cronback para cada subescala. O programa utilizado foi o Program Testat de Rudner adaptado por LORENZINI (1981).

\section{Coleta de dados}

A coleta de dados foi efetuada nos meses de setembro e outubro pela autora do trabalho.

\section{Análise dos dados}

Para a análise dos dados foi elaborado por Almeida Cunha um programa FORTRAN que fornece os escores médios das variáveis por indivíduo. Para a classificação dos sujeitos em relação à importância das variáveis foram estabelecidos escores médios que variavam de 3,00 - correspondente à opção "Sim" — até menor que um, correspondente à opção "não sei".

\section{RESULTADOS}

Em relação às características sócio-demográficas dos oitenta sujeitos da amostra, $68,7 \%$ tinham até 29 anos; 76,2\% eram casados; $60,0 \%$ possuíam até o ginasial incompleto e $68,7 \%$ possuíam renda mensal familiar até 4,5 salários mínimos:

\section{Classificação dos sujeitos em relação à importância das Variáveis do Modelo de Crenças em Saúde (MCS)}

\section{Suscetibilidade percebida}

$\mathrm{Na}$ Tabela 1 apresenta-se a distribuição das respostas dos sujeitos em relação à importância atribuída à variável do MCS - suscetibilidade percebida, $97,6 \%$ dos sujeitos são classificados no nível de muita e média importância, ou seja, as mães acham que os filhos são suscetíveis de contrair a difteria, coqueluche, tétano e poliomielite. Observa-se que os dados encontrados estão coincidentes com previsão do MCS e que resultados semelhantes foram encontrados por KEGELES (1963); STILLMAN (1977); BARRET (1979), em estudos realizados em outro país.

Assim sendo, na realidade local, ficou demonstrada a importância atribuída a esta variável do MCS pelos sujeitos deste estudo.

TABELA 1 - Distribuição do número e percentagem dos sujeitos da amostra seaundo a importância atribuída à variável suscetibilidade percebida.

\begin{tabular}{lrr}
\hline Importância atribuída à variável & N. & $\%$ \\
\hline Sem condições de opinar & 1 & 1,2 \\
Pouca & 1 & 1,2 \\
Média & 30 & 37,6 \\
Muita & 48 & 60,0 \\
\hline Total & 80 & 100,0 \\
\hline
\end{tabular}

\section{Gravidade percebida}

Em relação à importância atribuída à variável do MCS, gravidade percebida, observase na Tabela 2, que $98,0 \%$ das respostas dos sujeitos foram classificadas no nível de muita 
TABELA 2 - Distribuição do número e percentagem dos sujeitos da amostra segundo a importância atribuída à variável gravidade percebida.

\begin{tabular}{lrr}
\hline Importância da variável & N. & $\%$ \\
\hline Sem condições de opinar & 1 & 1,2 \\
Pouca & - & - \\
Média & $\overline{79}$ & 98,8 \\
Muita & 80 & 100,0 \\
\hline Total & \\
\hline \hline
\end{tabular}

TABELA 3 - Distribuição do número e percentagem dos sujeitos da amostra segundo a importância atribuída à variável benefícios percebidos.

\begin{tabular}{lrr}
\hline Importância da variável & N. & $\%$ \\
\hline Sem condição de opinar & 2 & 2,5 \\
Pouca & - & $-11,2$ \\
Média & 69 & 86,3 \\
Muita & 80 & 100,0 \\
\hline Total & 80 \\
\hline
\end{tabular}

importância, sugerindo que as mães percebem a difteria, coqueluche, tétano e poliomielite como graves.

Os dados encontrados com relação à essa variável são coincidentes com previsão do MCS e com resultados dos trabalhos de KEGELES (1966) e BARRET (1979).

\section{Beneficios percebidos}

$\mathrm{Na}$ Tabela 3 identifica-se a distribuição dos sujeitos em relação à importância atribuída à variável, benefícios percebidos para o ato de vacinar; $86,3 \%$ das respostas dos sujeitos são classificadas no nível de muita importância sugerindo que as mães percebem benefícios resultantes da aplicação das vacinas.

\section{Eventos motivadores}

Os dados da Tabela 4 mostram a distribuição dos sujeitos com relação à importância atribuída à variável eventos motivadores. Notase que os percentuais relativos aos níveis de muita e média importância, são bastante aproximados, não se podendo identificar predominância de um ou de outro nível.

Muitas mães afirmaram durante a eritrevista que o maior incentivo para o fato de estar vacinando o filho provinha geralmente da experiência positiva que possuía com outros filhos vacinados. Algumas mães também identificavam os conhecimentos adquiridos na escola, e outras com nível mais elevado de escolaridade reconheciam a vacina como algo que fazia
TABELA 4 - Distribuição do número e percentagem dos sujeitos da amostra segundo a importân. cia atribuída à variável eventos motivados.

\begin{tabular}{lrr}
\hline Importâncias das variáveis & $\mathrm{N}$. & $\%$ \\
\hline Sem condições de opinar & 2 & 2,5 \\
Pouca & 1 & 1,3 \\
Média & 38 & 47,5 \\
Muita & 39 & 48,7 \\
\hline Total & 80 & 100,0 \\
\hline
\end{tabular}

parte da vida normal de toda criança. BARRET (1979) também encontra uma média baixa nesta variável para as pessoas que utilizavam as clínicas de diagnóstico precoce de câncer. Para ROSENSTOCK (1966), os eventos motivadores funcionam como a força capaz de direcionar a ação preventiva. A intensidade desta força varia com os níveis percebidos de gravidade, e suscetibilidade; quando estes são elevados, como é o caso no presente estudo, a força necessária para ocasionar a ação preventiva é mínima. Justifica-se desta forma a tendência desta variável.

\section{Barreiras percebidas}

Com relação à distribuição das respostas dos sujeitos segundo a importância atribuída à variável barreiras percebidas, observa-se na Tabela 5, que $90,0 \%$ das respostas foram classificadas no nível de pouca importância. Este resultado sugere que as mães não reconhecem as barreiras estabelecidas neste estudo como impeditivas para vacinarem seus filhos. Estes dados estão coincidentes com a previsão do MCS.

Segundo este, quando não existem barreiras, ou estas são percebidas como baixas, a ação preventiva poderá ser tomada. BARRET (1979) encontrou resultados semelhantes.

TABELA 5 - Distribuição do número e percentagem dos sujeitos da amostra segundo a importância atribuída à variável barreiras percebidas.

\begin{tabular}{lrr}
\hline Importância das variáveis & $\mathrm{N} .{ }^{\circ}$ & $\%$ \\
\hline Sem condições de opinar & 2 & 2,5 \\
Pouca & 72 & 90,0 \\
Média & 6 & 7,5 \\
Muita & - & - \\
\hline Total & 80 & 100,0 \\
\hline
\end{tabular}

FERREIRA, S. L. Beliefs of mothers about vaccination. Rev. Bras. Enf., Brasília, 37(2): 109-115, 1984. 


\section{REFERENCIAS}

BARRET, M. B. The health behavior of person utilizing diagnostic cancer screening. Chicago, 1979. $66 \mathrm{p}$. (A research paper presented to the graduate school, department of nursing in partial ful. fillment of the requirements for the degree master of science).

BECKER, E. A negação da morte "apud" SOUZA, M. L. Mortalidade materna em Florianópolis-SC 1975-1979: obituário hospitalar. São Paulo, 1982. 133 p. (Tese de doutoramento - Faculdade de Saúde Pública da USP).

BOSKOVITZ, E. P. et alii. Levantamento das características epidemiológicas do sarampo em São José do Rio Preto: Rev. Saúde Públ., São Paulo, 8: $181-6,1974$.

CANDEIAS, N. M. F. \& MARCONDES, R. S. Diagnóstico em educação em saúde: um modelo para analisar as relações entre atitudes e práticas na área da saúde pública. Rev. Saúde Públ., São Paulo, 13: 63-8, 1979.

CASTRO, A. L. A educação em saúde em relação à vacinação. In: FARHAT, C. K. Fundamentos $e$ prática das imunizações. São Paulo, Medisa, 1981. cap. 64, 712 p.

CENTRO DE DEFESA DA QUALIDADE DA VIDA. A situação da criança no Brasil. Rio de Janeiro, 1979. $55 \mathrm{p}$.

COSTA, J. \& ALMEIDA, M. C. P. Orientações sobre vacinação infantil no momento da sua aplicação. Rev. Bras. Enf., Brasília, 33: 393-403, 1980.

CUMMINGS, K. M. et alii. Construct validation of the health belief model. Hlth. Educ. Monogr., Oakland, 6 (4): 394-405, 1978.

DIELMAN, T. E. et alii. Dimensions of children's health beliefs. Hlth. Educ. Quart., 7 (3): 218-238, 1980.

FERREIRA, C. E. Comportamento de professores e acadêmicos de odontologia acerca de práticas preventivas de saúde bucal. Florianópolis, 1981. (Dissertação de mestrado - Universidade Federal de Santa Catarina).

FISHBEIN, M. \& AJZEN, I. Belief, attitude, intention and behavior: an introduction to theory and research. Massachussets, Addison-Wesley Publishing, 1975. $578 \mathrm{p}$.

HALLAL, J. C. The relationships of health beliefs, health locus of control and self concept to the practice of breast self examination in adult human. Nurs. Res., New York, 31 (3): 137-42, 1982.

HOCHBAUM, G. M. Health behavior: basic concepts in health sciences series. Belmont, Wadsworth Publishing, 1974. $62 \mathrm{p}$.

JENKINS, D. C. The semantic differential for health: a technique for measuring. Publ. Hlth. Rep., Washington, 81 (6): 549-58, 1966.

KALS, S. V. et alii. Health behavior, illness behaivor ands ick-role behavior. Arch. Environ. Hlth., Chicago, 12: 531-41, Apr. 1966.

KEGELES, S. S. Some motives for seeking preven-
BIBLIOGRAFICAS

tive dental. J. Amer. Dent. Ass., Chicago, 67 (1): 90-8, 1963.

KIRSCHT, J. P. et alii. A national study of health beliefs. J. Hlth. Hum. Behav., Fort Whorth, 7: 248-54, 1966.

LORENZINI, F. Programa computacional para o cálculo do coeficiente alfa de Cronbach. Florianópolis, 1981.

MAHLER, H. Vacinar e proteger a criança. Saúde Mundo, Genebra, 3: fev./mar. 1977.

MAIMAN, L. A. et alii. Scales for measuring health beliefs model dimensions: a test of predictive value, internal consistency and relationships among beliefs. Hlth. Educ. Monogr., Oakland, 5 (3): 215-30, 1977.

MARCONDES, R. S. \& SOSSAI, J. A. Educação em saúde pública: conceitos básicos. São Paulo, 1977. (mimeografado).

MELO, C. G. O sistema de saúde em crise. 2. ed. São Paulo, Cebes-Hucitec, 1981. 205 p.

MINISTERIO DA SAƯDE. Normas e condições para o desenvolvimento do programa nacional de imunizações. Bol. Epidem., Rio de Janeiro, 8 (25): 245-52, 1976.

- Programa Nacional de imunizações - resultados observados em 1977. Bol. Epidem., Rio de Janeiro, 12 (9): 77-80, 1980.

NUNNALY, I. C. Introducion a la medición psicologica. Buenos Aires, Centro Regional de Ayuda Técnica, 1964. $619 \mathrm{p}$.

OGUISSO, T. Repercussão do sistema nacional de saúde nos modelos assistenciais. Rev. Esc. Enf. USP, São Paulo, 14 (2): 147-64, 1980.

ORGANIZAÇAO MUNDIAL DE SAƯDE. Grupo científico sobre imunodeficiência. Washington, 1977. 32 p. (Informes Técnicos, 630).

ORGANIZAÇAO PANAMERICANA DA SAUUDE. $A$ saúde das crianças no mundo: necessidades $e$ problemas. Washington, 1979. 9 p. (Publicação do Ano Internacional da Criança, 3 ).

- Proteção de todas as crianças contra as doenças evitáveis. Washington, 1979. 5 p. (Publicação do Ano Internacional da Criança, 6).

ROSENSTOCK, I. M. Historical, origins of the health beliefs model. Hlth. Educ. Monogr., Oakland, 2 (4): 324-476, 1974.

- Why people use health services. Milbank Mem. Fd. Quart., New York, 64 (3): 94-127, 1966.

STILLMAN, M. Women's health beliefs about breast cancer and breast self examination. Nurs. Res., New York, 26 (2): 121-6, 1977.

WATTS, R. J. Sexual functioning, health beliefs and compliance with high blood pressure medications. Nurs. Res., New York, 31 (5): 278-283, 1982.

WESTRUP, M. H. B. et alii. Doenças preveníveis por vacinas em crianças de 0 a 12 anos, atendidas em serviços de saúde de Florianópolis, SC. In: CONGRESSO BRASILEIRO DE ENFERMAGEM, 35. 